\title{
STUDY OF THE BLOW UP OF THE MAXIMAL SOLUTION TO THE THREE-DIMENSIONAL MAGNETOHYDRODYNAMIC SYSTEM IN LEI-LIN-GEVREY SPACES
}

\section{RIDHA SELMI ${ }^{1,2,3, *}$, JAMEL BENAMEUR ${ }^{4}$}

${ }^{1}$ Department of Mathematics, College of Sciences, Northern Border University, P.O. Box 1321, Arar, 73222, KSA

${ }^{2}$ Faculty of Sciences of Gabes, University of Gabes, 6072, Gabès, Tunisia

${ }^{3}$ Laboratory of partial differential equations and applications (LR03ES04), Faculty of sciences of Tunis, University of Tunis El Manar, 1068 Tunis, Tunisia

${ }^{4}$ Department of Mathematics, ISSAT Gabès, University of Gabès, Tunisia

${ }^{*}$ Corresponding author: Ridha.selmi@nbu.edu.sa

\begin{abstract}
In this paper, we investigate existence, uniqueness and blowup in finite time of the local solution to the three dimensional magnetohydrodynamic system, in Gevrey-Lei-Lin spaces. To prove the blowup results and give the blow profile as a function of time, two key points are used. The first is a frequency decomposition of the spectrum of the initial data. This allows to use Leray theory. The second is a technical lemma we proved to state that the Lei-Lin space is an interpolation space between the Gevrey-Lei-Lin and the Lebesgue square integrable functions spaces. To prove uniqueness, we use a penalization procedure and energy methods. About existence, we split the initial condition into low frequencies part and high frequencies part. The former are considered as initial data to the linear part of the system. The latter will be taken as small as needed, so that smallness theory applies and allows to run a fixed point argument.
\end{abstract}

Received February $8^{\text {th }}, 2020$; accepted February $28^{\text {th }}, 2020$; published May $1^{\text {st }}, 2020$.

2010 Mathematics Subject Classification. 35A01, 35A02, 35B40, 35B44, 35B45.

Key words and phrases. magnetohydrodynamic system; critical spaces; existence; uniqueness; blowup; Gevrey-Lein-Lin space; frequency decomposition.

(C)2020 Authors retain the copyrights of their papers, and all open access articles are distributed under the terms of the Creative Commons Attribution License. 
Let us consider the following three dimensional incompressible magnetohydrodynamic system,

$$
\left\{\begin{array}{l}
\partial_{t} u-\Delta u+u \cdot \nabla u-b \cdot \nabla b+\nabla\left(p+\frac{1}{2}|b|^{2}\right)=0, \quad(t, x) \in \mathbb{R}^{+} \times \mathbb{R}^{3} \\
\partial_{t} b-\Delta b+u \cdot \nabla b-b \cdot \nabla u=0, \quad(t, x) \in \mathbb{R}^{+} \times \mathbb{R}^{3} \\
\operatorname{div} u=0, \quad(t, x) \in \mathbb{R}^{+} \times \mathbb{R}^{3} \\
\operatorname{div} b=0, \quad(t, x) \in \mathbb{R}^{+} \times \mathbb{R}^{3} \\
(u, b)(0)=\left(u^{0}, b^{0}\right), \quad x \in \mathbb{R}^{3},
\end{array}\right.
$$

where $u, b$ and $p$ denote respectively the unknown velocity, the unknown magnetic field and the unknown pressure at the point $(t, x)$. If the initial data $u^{0}$ and $b^{0}$ are quite regular, the divergence free conditions determine the pressure $p$. We aim to study the existence, uniqueness and blowup in finite time of local solution to the MHD system, in the framework of Gevrey-Lei-Lin spaces. These spaces are defined for the real numbers $a>0, \sigma>1$ and $\rho$, by

$$
\mathcal{X}_{a, \sigma}^{\rho}\left(\mathbb{R}^{3}\right)=\left\{f \in \mathcal{S}^{\prime}\left(\mathbb{R}^{3}\right) ; \widehat{f} \in L_{l o c}^{1}\left(\mathbb{R}^{3}\right), \int_{\mathbb{R}^{3}}|\xi|^{\rho} e^{a|\xi|^{1 / \sigma}}|\hat{f}(\xi)| d \xi<\infty\right\}
$$

and endowed with its naturel norm

$$
\|f\|_{\mathcal{X}_{a, \sigma}^{\rho}\left(\mathbb{R}^{3}\right)}=\int_{\mathbb{R}^{3}}|\xi|^{\rho} e^{a|\xi|^{1 / \sigma}}|\hat{f}(\xi)| d \xi
$$

where $\hat{f}$ denotes the Fourier transform of $f$.

In [15], the authors defined the Lei-Lin space by

$$
\mathcal{X}^{-1}\left(\mathbb{R}^{3}\right)=\left\{f \in \mathcal{S}^{\prime}\left(\mathbb{R}^{3}\right), \widehat{f} \in L_{l o c}^{1}\left(\mathbb{R}^{3}\right), \int_{\mathbb{R}^{3}} \frac{|\widehat{f}(\xi)|}{|\xi|} d \xi<\infty\right\}
$$

and endowed with its natural norm

$$
\|f\|_{\mathcal{X}^{-1}\left(\mathbb{R}^{3}\right)}=\int_{\mathbb{R}^{3}} \frac{|\hat{f}(\xi)|}{|\xi|} d \xi
$$

Here, $L_{l o c}^{1}\left(\mathbb{R}^{3}\right)$ states for the set of locally $\mathbb{R}^{3}$-Lebesgue integrable distributions. In this critical space, the distinguishable fact was that to obtain global well-posedness to the Navier-Stokes equations, the norm of the initial data have to be exactly less than the viscosity of the fluid. However, in the wide fluid mechanic literature, it was always assumed that the initial data must be very small, especially smaller than the viscosity multiplied by a tiny positive constant. Such assumption is mandatory to run the smallness argument and to obtain global well-posedness; see for example [11-14] and a complete survey in [10]. For many fluid mechanics equations, well-posedness and asymptotic behavior, as time goes to infinity or as small parameter goes to zero, were investigated by the authors, in various spaces; see for example [6-9, 18-20]. About blowup, it is worthwhile to emphasize that several authors studied this phenomena to the Navier-Stokes equations; see for example $[1-3,17]$ and references therein. The author observed, in [4], that in the case of the Navier-Stokes equations, the blowup phenomena depends on the chosen space not on the nonlinear part. To do so, he used Fourier analysis in Sobolev-Gevrey spaces, for 
Sobolev index $s>3 / 2$. His blowup result was improved later on, in [5], where the authors gave precisely an exponential type of the blowup profile, in Sobolev-Gevrey spaces but with less regularity on the initial data since they dealt with $s=1$. In [16], authors studied the Cauchy problem for a two-components high-order Camassa-Holm system. First, they proved the local well-posedness of the system in Besov spaces. Then, using Littlewood-Paley theory, they derived a blowup criterion for the strong solution. Finally, they studied Gevrey regularity and analyticity of the solutions to the Camassa-Holm system in the Gevrey-Sobolev spaces.

In this paper, we begin by addressing the problem of local well-posedness. Our result is summarized in the following existence and uniqueness theorem.

Theorem 0.1. Let $\left(u^{0}, b^{0}\right) \in\left(\mathcal{X}_{a, \sigma}^{-1}\left(\mathbb{R}^{3}\right)\right)^{2}$. Then, there exist a time $T>0$ and a unique solution $(u, b) \in$ $\mathcal{C}\left([0, T],\left(\mathcal{X}_{a, \sigma}^{-1}\left(\mathbb{R}^{3}\right)\right)^{2}\right)$ of $(M H D)$, such that $(u, b) \in L^{1}\left([0, T],\left(\mathcal{X}_{a, \sigma}^{1}\left(\mathbb{R}^{3}\right)\right)^{2}\right)$.

To prove uniqueness of solution, we use a penalisation method. This allows to put the problem in a form where Gronwall inequality can be applied. To establish existence of solution, we split the initial condition into low and high frequencies. The former will be considered as initial data to the linear part of the $M H D$ system. The latter, taken as small as needed, will be the initial data to the remaining nonlinear part, for which smallness theory applies and allows to run a fixed point argument, in $\left(\mathcal{X}_{a, \sigma}^{-1}\left(\mathbb{R}^{3}\right)\right)^{2}$.

Then, we turn to the blowup result that we state in the following theorem.

Theorem 0.2. Let $(u, b) \in C\left(\left[0, T^{*}\left[,\left(\mathcal{X}_{a, \sigma}^{-1}\left(\mathbb{R}^{3}\right)\right)^{2}\right)\right.\right.$ be the maximal solution of MHD system, where $T^{*}<\infty$. Then, there exists $c_{0}>0$, such that

$$
\liminf _{t \nearrow T^{*}}\left(T^{*}-t\right)^{1 / 3} e^{-c_{0}\left(T^{*}-t\right)^{-\frac{1}{3 \sigma}}}\|(u, b)(t)\|_{\mathcal{X}_{a, \sigma}^{-1}} \geq \frac{1}{2} .
$$

The proof is somewhat technical. Our idea is to use a suitable frequency decomposition and to impose "the problematic" large frequencies part to be a square integrable function, so that we fully profit from Leray theory. Some technical lemmas are specially derived to handle technical difficulties, mainly Lemma 1.1, where we proved that $\mathcal{X}^{0}$ is an interpolation space between the Lei-Lin spaces $\mathcal{X}^{s}$ and the space of Lebesgue square integrable functions. The structure of our proof is as follows. Starting with the energy estimates, we prove that the $\mathcal{X}_{a, \sigma}^{-1}$ norm of the solution blows up, in finite time. Gronwall type inequality allows to infer that blowup holds also in $\mathcal{X}_{a^{\prime}, \sigma}^{0}$, for any $a^{\prime} \in(0, a)$. Using a particular choice of parameter $a^{\prime}$, we deduce that our solution blows up in the $\mathcal{X}^{0}$ norm, as a limit of $\mathcal{X}_{a^{\prime}, \sigma}^{0}$ spaces. We split the initial data into two parts, a large frequencies one that belongs to $\mathcal{X}_{a, \sigma}^{-1} \cap L^{2}$ and a small frequencies remainder that leis in $\mathcal{X}_{a, \sigma}^{-1}$. The smallness theory applies an leads to a global and continuous in time solution that belongs to $\mathcal{X}_{a, \sigma}^{-1}$. This continuity plays an important role. About the large frequencies part, the $L^{2}$ theory applies and allows to derive a Leray type energy estimates. We use the above two estimates to dominate the $\mathcal{X}^{0}$ norm 
of the solution. Finally, Lemma 1.1 with a judicious choice of the index $s$, finish the proof of the blowup result, in $\mathcal{X}_{a, \sigma}^{-1}$ and determine its profile as a function of time.

This paper is organized as follows. In section 2, we give some notations and useful preliminary results. Section 3 is devoted to prove existence and uniqueness of local in time solution. In section 4, we establish the blowup result.

\section{TeChnical Lemmas}

In this section, we prove some technical lemmas that will be used later on.

Lemma 1.1. Let $s \geq 3 / 2$, there exists $C>0$ such that, $\forall f \in L^{2}\left(\mathbb{R}^{3}\right) \cap \mathcal{X}^{s}\left(\mathbb{R}^{3}\right)$,

$$
\|f\|_{\mathcal{X}^{0}} \leq C\|f\|_{L^{2}}^{\frac{s}{s+\frac{3}{2}}}\|f\|_{\mathcal{X}^{s}}^{\frac{\frac{3}{2}}{s+\frac{3}{2}}}
$$

Proof. For $\lambda>0$, we consider the decomposition

$$
\|f\|_{\mathcal{X}^{0}}=\underbrace{\int_{|\xi|<\lambda}|\widehat{f}(\xi)| d \xi}_{I(\lambda)}+\underbrace{\int_{|\xi|>\lambda}|\widehat{f}(\xi)| d \xi}_{J(\lambda)} .
$$

By Cauchy-Schwarz inequality for $I(\lambda)$ and direct computation for $J(\lambda)$, we get

$$
\begin{aligned}
I(\lambda) & \leq \sqrt{4 \pi} \lambda^{3 / 2}\|f\|_{L^{2}}, \\
J(\lambda) & \leq \lambda^{-s}\|f\|_{\mathcal{X}^{s}} .
\end{aligned}
$$

For $A=\sqrt{4 \pi}\|f\|_{L^{2}}$ and $B=\|f\|_{\mathcal{X}^{s}}$, let $\varphi(\lambda)=A \lambda^{3 / 2}+B \lambda^{-s}$. Clearly the value $\lambda_{0}=\left(\frac{2 s B}{3 A}\right)^{\frac{1}{s+\frac{3}{2}}}$ is a minimum of $\varphi$ and for all $s \geq 3 / 2, \varphi\left(\lambda_{0}\right) \leq C A^{\frac{s}{s+\frac{3}{2}}} B^{\frac{3 / 2}{s+\frac{3}{2}}}$.

Lemma 1.2. Let $\sigma>1$, then there is a constant $C>0$, such that for all $f, g \in \mathcal{X}_{a, \sigma}^{-1}\left(\mathbb{R}^{3}\right)$, we have

$$
\|f g\|_{\mathcal{X}_{a, \sigma}^{0}} \leq C\|f\|_{\mathcal{X}_{a, \sigma}^{0}}\|g\|_{\mathcal{X}_{\frac{a}{\sigma}, \sigma}^{0}}+C\|g\|_{\mathcal{X}_{a, \sigma}^{0}}\|f\|_{\mathcal{X}_{\frac{a}{\sigma}, \sigma}^{0}}
$$

Proof. In a first step, using the triangular inequality and the fact that

$$
|\xi| \leq \max (|\xi-\eta|,|\eta|)\left(1+\frac{\min (|\xi-\eta|,|\eta|)}{\max (|\xi-\eta|,|\eta|)}\right)
$$

we obtain

$$
\|f g\|_{\mathcal{X}_{a, \sigma}^{0}} \leq \int_{\xi} \int_{\eta} e^{a \max (|\xi-\eta|,|\eta|)^{1 / \sigma}\left(1+\frac{\min (|\xi-\eta|,|\eta|)}{\max (|\xi-\eta|,|\eta|)}\right)^{1 / \sigma}}|\widehat{f}(\xi-\eta)| \cdot|\widehat{g}(\eta)| d \eta d \xi
$$

In a second step, the inequality $\left.\left.(1+r)^{\theta} \leq 1+\theta r^{\theta}, \forall r \in[0,1], \forall \theta \in\right] 0,1\right]$ leads to

$$
a \max (|\xi-\eta|,|\eta|)^{1 / \sigma}\left(1+\frac{\min (|\xi-\eta|,|\eta|)}{\max (|\xi-\eta|,|\eta|)}\right)^{1 / \sigma} \leq a \max (|\xi-\eta|,|\eta|)^{1 / \sigma}+\frac{a}{\sigma} \min (|\xi-\eta|,|\eta|)^{1 / \sigma} .
$$

Finally, distinguishing the two cases $|\xi-\eta|<|\eta|$ and $|\xi-\eta|>|\eta|$, we obtain the desired result. 
By Cauchy-Schwarz inequality, we prove the following lemma.

Lemma 1.3. For all $f \in \mathcal{X}_{a, \sigma}^{s-1}\left(\mathbb{R}^{3}\right) \cap \mathcal{X}_{a, \sigma}^{s+1}\left(\mathbb{R}^{3}\right)$, with $\sigma>1$ and $a>0$, we have

$$
\|f\|_{\mathcal{X}_{a, \sigma}^{s}} \leq\|f\|_{\mathcal{X}_{a, \sigma}^{s-1}}^{1 / 2}\|f\|_{\mathcal{X}_{a, \sigma}^{s+1}}^{1 / 2}
$$

Using the fact that $|\xi|^{1 / \sigma} \leq(|\xi-\eta|+|\eta|)^{1 / \sigma} \leq|\xi-\eta|^{1 / \sigma}+|\eta|^{1 / \sigma}$, we have the lemma below.

Lemma 1.4. For all $f \in \mathcal{X}_{a, \sigma}^{0}\left(\mathbb{R}^{3}\right), g \in \mathcal{X}_{a, \sigma}^{1}\left(\mathbb{R}^{3}\right)$, with $\sigma>1$ and $a>0$, we have

$$
\|f . \nabla g\|_{\mathcal{X}_{a, \sigma}^{0}} \leq\|f\|_{\mathcal{X}_{a, \sigma}^{0}}\|g\|_{\mathcal{X}_{a, \sigma}^{1}}
$$

Lemma 1.5. Let $u, v \in L^{\infty}\left([0, T], \mathcal{X}_{a, \sigma}^{-1}\left(\mathbb{R}^{3}\right)\right) \cap L^{1}\left([0, T], \mathcal{X}_{a, \sigma}^{1}\left(\mathbb{R}^{3}\right)\right)$. Then

$$
\left\|\int_{0}^{t} e^{(t-\tau) \Delta} \operatorname{div}(u v) d \tau\right\|_{\mathcal{X}_{a, \sigma}^{-1}} \leq\|u\|_{L_{T}^{\infty}\left(\mathcal{X}_{a, \sigma}^{-1}\right)}^{1 / 2}\|u\|_{L_{T}^{1}\left(\mathcal{X}_{a, \sigma}^{1}\right)}^{1 / 2}\|v\|_{L_{T}^{\infty}\left(\mathcal{X}_{a, \sigma}^{-1}\right)}^{1 / 2}\|v\|_{L_{T}^{1}\left(\mathcal{X}_{a, \sigma}^{1}\right)}^{1 / 2} .
$$

Proof. First of all, let us prove that for $f, g \in \mathcal{X}_{a, \sigma}^{-1}\left(\mathbb{R}^{3}\right) \cap \mathcal{X}_{a, \sigma}^{1}\left(\mathbb{R}^{3}\right)$, we have

$$
\|f g\|_{\mathcal{X}_{a, \sigma}^{0}} \leq\|f\|_{\mathcal{X}_{a, \sigma}^{-1}}^{1 / 2}\|f\|_{\mathcal{X}_{a, \sigma}^{1}}^{1 / 2}\|g\|_{\mathcal{X}_{a, \sigma}^{-1}}^{1 / 2}\|g\|_{\mathcal{X}_{a, \sigma}^{1 / 2}}^{1 / 2}
$$

To do so, we recall that

$$
\|f g\|_{\mathcal{X}_{a, \sigma}^{0}} \leq \int_{\xi} e^{a|\xi|^{1 / \sigma}}\left(\int_{\eta}|\hat{f}(\xi-\eta)||\hat{g}(\eta)| d \eta\right) d \xi
$$

Using the inequality $e^{a|\xi|^{1 / \sigma}} \leq e^{a|\xi-\eta|^{1 / \sigma}} e^{a|\eta|^{1 / \sigma}}$, we obtain

$$
\|f g\|_{\mathcal{X}_{a, \sigma}^{0}} \leq \int_{\xi}\left(\int_{\eta} e^{a|\xi-\eta|^{1 / \sigma}}|\hat{f}(\xi-\eta)| \cdot e^{a|\eta|^{1 / \sigma}} \cdot|\hat{g}(\eta)| d \eta\right) d \xi .
$$

Put $F(\xi)=e^{a|\xi|^{1 / \sigma}}|\hat{f}(\xi)|$ and $G(\xi)=\frac{e^{a|\xi|^{1 / \sigma}}}{|\xi|}|\hat{f}(\xi)|$. It holds that,

$$
\begin{aligned}
\|f g\|_{\mathcal{X}_{a, \sigma}^{0}} & \leq\|F * G\|_{L^{1}} \\
& \leq\|F\|_{L^{1}}\|G\|_{L^{1}} \\
& \leq\|f\|_{\mathcal{X}_{a, \sigma}^{0}}\|g\|_{\mathcal{X}_{a, \sigma}^{0}} .
\end{aligned}
$$

By Cauchy-Schwarz inequality, we get (1.1). To continue proving lemma above, we have

$$
\begin{array}{r}
\int_{0}^{t}\left\|e^{(t-\tau) \Delta} \operatorname{div}(u v) d \tau\right\|_{\mathcal{X}_{a, \sigma}^{-1}} \leq\left\|\int_{0}^{t} e^{(t-\tau) \Delta} \operatorname{div}(u v)\right\|_{\mathcal{X}_{a, \sigma}^{-1}} d \tau \\
\left.\leq \int_{0}^{t} \| u v\right) \|_{\mathcal{X}_{a, \sigma}^{0}} d \tau \\
\leq \int_{0}^{t}\|u\|_{\mathcal{X}_{a, \sigma}^{0}}\|v\|_{\mathcal{X}_{a, \sigma}^{0}} d \tau \\
\leq \int_{0}^{t}\|u\|_{\mathcal{X}_{a, \sigma}^{-1}}^{1 / 2}\|u\|_{\mathcal{X}_{a, \sigma}^{1}}^{1 / 2}\|v\|_{\mathcal{X}_{a, \sigma}^{-1}}^{1 / 2}\|v\|_{\mathcal{X}_{a, \sigma}^{1}}^{1 / 2} d \tau \\
\leq\|u\|_{L_{T}^{\infty}\left(\mathcal{X}_{a, \sigma}^{-1}\right)}^{1 / 2}\|v\|_{L_{T}^{\infty}\left(\mathcal{X}_{a, \sigma}^{-1}\right)}^{1 / 2} \int_{0}^{T}\|u\|_{\mathcal{X}_{a, \sigma}^{1}}^{1 / 2}\|v\|_{\mathcal{X}_{a, \sigma}^{1}}^{1 / 2} d \tau \\
\leq\|u\|_{L_{T}^{\infty}\left(\mathcal{X}_{a, \sigma}^{-1}\right)}^{1 / 2}\|v\|_{L_{T}^{\infty}\left(\mathcal{X}_{a, \sigma}^{-1}\right)}^{1 / 2}\|u\|_{L_{T}^{1}\left(\mathcal{X}_{a, \sigma}^{1}\right)}^{1 / 2}\|v\|_{L_{T}^{1}\left(\mathcal{X}_{a, \sigma}^{1}\right)}^{1 / 2}
\end{array}
$$


Lemma 1.6. Let $u, v \in L_{T}^{\infty}\left(\mathcal{X}_{a, \sigma}^{-1}\left(\mathbb{R}^{3}\right)\right) \cap L_{T}^{1}\left(\mathcal{X}_{a, \sigma}^{1}\left(\mathbb{R}^{3}\right)\right)$. Then

$$
\left\|\int_{0}^{t} e^{(t-\tau) \Delta} \operatorname{div}(u v) d \tau\right\|_{L_{T}^{1}\left(\mathcal{X}_{a, \sigma}^{1}\right)} \leq\|u\|_{L_{T}^{\infty}\left(\mathcal{X}_{a, \sigma}^{-1}\right)}^{1 / 2}\|v\|_{L_{T}^{\infty}\left(\mathcal{X}_{a, \sigma}^{-1}\right)}^{1 / 2}\|u\|_{L_{T}^{1}\left(\mathcal{X}_{a, \sigma}^{1}\right)}^{1 / 2}\|v\|_{L_{T}^{1}\left(\mathcal{X}_{a, \sigma}^{1}\right)}^{1 / 2}
$$

Proof. Using the definition of $\mathcal{X}_{a, \sigma}^{1}$ norm and integrating the function $e^{-(t-\tau)|\xi|^{2}}$ twice with respect to $\tau \in[0, t]$ and $t \in[0, T]$, it follows that

$$
\begin{array}{r}
\int_{0}^{T}\left\|\int_{0}^{t} e^{(t-\tau) \Delta} \operatorname{div}(u v) d \tau\right\|_{\mathcal{X}_{a, \sigma}^{1}} d t \\
\leq \int_{0}^{T} \int_{0}^{t} \int_{\mathbb{R}^{3}} e^{-(t-\tau)|\xi|^{2}}|\xi|^{2} e^{a|\xi|^{1 / \sigma}}|\widehat{u v}(\tau, \xi)| d \tau d t d \xi \\
\leq \int_{\mathbb{R}^{3}}|\xi|^{2} e^{a|\xi|^{1 / \sigma}}\left(\int_{0}^{T} \int_{0}^{t} e^{-(t-\tau)|\xi|^{2}}|\widehat{u v}(\tau, \xi)| d \tau d t\right) d \xi \\
\leq \int_{\mathbb{R}^{3}}|\xi|^{2} e^{a|\xi|^{1 / \sigma}}\left(\int_{0}^{T}|\widehat{u v}(\tau, \xi)|\left(\left[\frac{-e^{-(t-\tau)|\xi|^{2}}}{|\xi|^{2}}\right]_{\tau}^{T}\right) d \tau\right) d \xi \\
\leq \int_{\mathbb{R}^{3}}|\xi|^{2} e^{a|\xi|^{1 / \sigma}}\left(\int_{0}^{T}|\widehat{u v}(\tau, \xi)|\left(\frac{1-e^{-(T-\tau)|\xi|^{2}}}{|\xi|^{2}}\right) d \tau\right) d \xi \\
\leq \int_{0}^{T}\|u v\|_{\mathcal{X}_{a, \sigma}^{0}} d \tau .
\end{array}
$$

Using equation (1.1) finishes the proof.

\section{WeLl-POSEDNESS RESUlts}

To prove uniqueness of solution to the $(M H D)$, we consider two solutions $\left(u_{1}, b_{1}\right)$ and $\left(u_{2}, b_{2}\right)$ that belong to $\left(\mathcal{C}\left([0, T], \mathcal{X}_{a, \sigma}^{-1}\left(\mathbb{R}^{3}\right)\right)\right)^{2} \cap\left(L^{1}\left([0, T], \mathcal{X}_{a, \sigma}^{1}\left(\mathbb{R}^{3}\right)\right)\right)^{2}$ and have the same initial data. Let $\delta=u_{1}-u_{2}$ and $\eta=b_{1}-b_{2}$, it follows that

$$
\left\{\begin{array}{l}
\partial_{t} \delta-\Delta \delta+\delta \cdot \nabla u_{2}+u_{1} \cdot \nabla \delta-\eta \cdot \nabla b_{2}-b_{1} \cdot \nabla \eta+\nabla\left(p_{1}-p_{2}+\frac{1}{2}\left(\left|b_{1}\right|^{2}-\left|b_{2}\right|^{2}\right)\right)=0 \\
\partial_{t} \eta-\Delta \eta+u_{2} \cdot \nabla \eta-\eta \cdot \nabla u_{1}+\delta \cdot \nabla b_{1}-b_{2} \cdot \nabla \delta=0 \\
\operatorname{div} \delta=0 \\
\operatorname{div} \eta=0 \\
(\delta, \eta)=(0,0) .
\end{array}\right.
$$

Taking the Fourier transform and using divergence free condition, one infers that

$$
\begin{array}{r}
\partial_{t} \hat{\delta}+|\xi|^{2} \hat{\delta}+\left(\widehat{\delta \cdot \nabla u_{2}}\right)+\left(\widehat{u_{1} \cdot \nabla} \delta\right)-\left(\widehat{\eta \cdot \nabla b_{2}}\right)-\left(\widehat{b_{1} \cdot \nabla \eta}\right)=0 \\
\partial_{t} \hat{\eta}+|\xi|^{2} \hat{\eta}+\left(\widehat{u_{2} \cdot \nabla \eta}\right)-\left(\widehat{\eta \cdot \nabla u_{1}}\right)+\left(\widehat{\delta \cdot \nabla b_{1}}\right)-\left(\widehat{b_{2} \cdot \nabla} \delta\right)=0 .
\end{array}
$$

Multiplying (2.2) (respectively (2.3)) by $\overline{\hat{\delta}}$ (respectively $\overline{\hat{\eta}}$ ) and its conjugate by $\hat{\delta}$ (respectively $\hat{\eta}$ ), summing up the four resulting equations, and dominating the real part of any complex quantity by its modulus, we 
obtain

$$
\begin{aligned}
\frac{1}{2} \partial_{t}\left(|\hat{\delta}|^{2}\right. & \left.+|\hat{\eta}|^{2}\right)+|\xi|^{2}\left(|\hat{\delta}|^{2}+|\hat{\eta}|^{2}\right) \\
& \leq\left|\left(\widehat{\delta . \nabla u_{2}}\right)\right||\overline{\hat{\delta}}|+\left|\left(\widehat{u_{1} \cdot \nabla \delta}\right)\right||\overline{\hat{\delta}}|+\left|\left(\widehat{\eta \cdot \nabla b_{2}}\right)\right||\overline{\hat{\delta}}|+\left|\left(\widehat{b_{1} \cdot \nabla \eta}\right)\right||\overline{\hat{\delta}}| \\
& +\left|\left(\widehat{u_{2} \cdot \nabla \eta}\right)\right||\overline{\hat{\eta}}|+\left|\left(\widehat{\eta \cdot \nabla u_{1}}\right)\right||\overline{\hat{\eta}}|+2\left|\left(\widehat{\delta . \nabla b_{1}}\right)\right||\overline{\hat{\eta}}|+\left|\left(\widehat{b_{2} \cdot \nabla \delta}\right)\right||\overline{\hat{\eta}}| .
\end{aligned}
$$

Let $\varepsilon>0$ be a penalizing parameter. One has

$$
\partial_{t}\left(|\hat{\delta}|^{2}+|\hat{\eta}|^{2}\right)=\partial_{t}\left(|\hat{\delta}|^{2}+|\hat{\eta}|^{2}+\varepsilon^{2}\right)=2 \sqrt{|\hat{\delta}|^{2}+|\hat{\eta}|^{2}+\varepsilon^{2}} \cdot \partial_{t} \sqrt{|\hat{\delta}|^{2}+|\hat{\eta}|^{2}+\varepsilon^{2}}
$$

Substituting (2.5) in (2.4), dividing by $\sqrt{|\hat{\delta}|^{2}+|\hat{\eta}|^{2}+\varepsilon^{2}}$, integrating with respect to time, letting $\varepsilon \rightarrow 0$ and using that $\frac{|\hat{\delta}|+|\hat{\eta}|}{\sqrt{2}} \leq \sqrt{|\hat{\delta}|^{2}+|\hat{\eta}|^{2}}$, we infer that

$$
\begin{aligned}
|\hat{\delta}|+|\hat{\eta}| & +\int_{0}^{t}|\xi|^{2}(|\hat{\delta}|+|\hat{\eta}|) d \tau \\
& \leq \sqrt{2}\left(\int_{0}^{t}\left(\left|\left(\widehat{\delta . \nabla u_{2}}\right)\right||\overline{\hat{\delta}}|+\left|\left(\widehat{u_{1} \cdot \nabla \delta}\right)\right||\hat{\hat{\delta}}|+\left|\left(\widehat{\eta \cdot \nabla b_{2}}\right)\right||\overline{\hat{\delta}}|+\left|\left(\widehat{b_{1} \cdot \nabla \eta}\right)\right||\overline{\hat{\delta}}|\right) d \tau\right. \\
& +\int_{0}^{t}\left(\left|\left(\widehat{u_{2} \cdot \nabla \eta}\right)\right||\overline{\hat{\eta}}|+\left|\left(\widehat{\eta \cdot \nabla u_{1}}\right)\right||\overline{\hat{\eta}}|+2\left|\left(\widehat{\delta \cdot \nabla b_{1}}\right)\right||\overline{\hat{\eta}}|+\left|\left(\widehat{b_{2} \cdot \nabla \delta}\right)\right||\overline{\hat{\eta}}| d \tau\right) .
\end{aligned}
$$

Multiplying by $\frac{e^{a|\xi| \frac{1}{\sigma}}}{|\xi|}$ and integrating with respect to $\xi$. By divergence free, we get

$$
\begin{aligned}
\|\delta\|_{\mathcal{X}_{a, \sigma}^{-1}}+\|\eta\|_{\mathcal{X}_{a, \sigma}^{-1}} & +\int_{0}^{t}\left(\|\Delta \delta\|_{\mathcal{X}_{a, \sigma}^{-1}}+\|\Delta \eta\|_{\mathcal{X}_{a, \sigma}^{-1}}\right) d \tau \\
& \leq \sqrt{2}\left(\int_{0}^{t}\left\|\delta u_{2}\right\|_{\mathcal{X}_{a, \sigma}^{0}}+\left\|u_{1} \delta\right\|_{\mathcal{X}_{a, \sigma}^{0}}+\left\|\eta b_{2}\right\|_{\mathcal{X}_{a, \sigma}^{0}}+\left\|b_{1} \eta\right\|_{\mathcal{X}_{a, \sigma}^{0}} d \tau\right. \\
& \left.+\int_{0}^{t}\left\|u_{2} \eta\right\|_{\mathcal{X}_{a, \sigma}^{0}}+\left\|\eta u_{1}\right\|_{\mathcal{X}_{a, \sigma}^{0}}+\left\|\delta b_{1}\right\|_{\mathcal{X}_{a, \sigma}^{0}}+\left\|b_{2} \delta\right\|_{\mathcal{X}_{a, \sigma}^{0}} d \tau\right) .
\end{aligned}
$$

Using the product Young inequality, it follows that

$$
\begin{aligned}
\left\|\delta u_{2}\right\|_{\mathcal{X}_{a, \sigma}^{0}} & \leq\|\delta\|_{\mathcal{X}_{a, \sigma}^{0}}\left\|u_{2}\right\|_{\mathcal{X}_{a, \sigma}^{0}} \\
& \leq\|\delta\|_{\mathcal{X}_{a, \sigma}^{-1}}^{\frac{1}{2}}\|\Delta \delta\|_{\mathcal{X}_{a, \sigma}^{-1}}^{\frac{1}{2}}\left\|u_{2}\right\|_{\mathcal{X}_{a, \sigma}^{-1}}^{\frac{1}{2}}\left\|\Delta u_{2}\right\|_{\mathcal{X}_{a, \sigma}^{-1}}^{\frac{1}{2}} \\
& \leq 2\|\delta\|_{\mathcal{X}_{a, \sigma}^{-1}}\left\|u_{2}\right\|_{\mathcal{X}_{a, \sigma}^{-1}}\left\|\Delta u_{2}\right\|_{\mathcal{X}_{a, \sigma}^{-1}}+\frac{1}{2}\|\Delta \delta\|_{\mathcal{X}_{a, \sigma}^{-1}},
\end{aligned}
$$

and so on for the other terms. Thus,

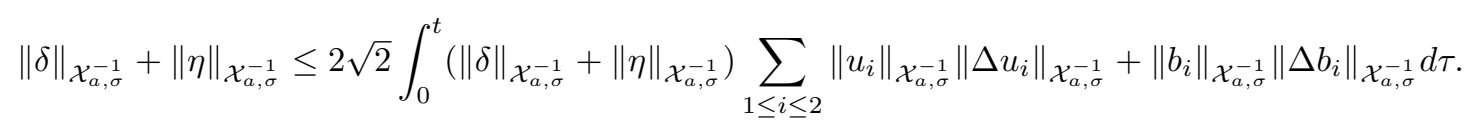

Since the function $t \mapsto \sum_{1 \leq i \leq 2}\left\|u_{i}\right\|_{\mathcal{X}_{a, \sigma}^{-1}}\left\|\Delta u_{i}\right\|_{\mathcal{X}_{a, \sigma}^{-1}}+\left\|b_{i}\right\|_{\mathcal{X}_{a, \sigma}^{-1}}\left\|\Delta b_{i}\right\|_{\mathcal{X}_{a, \sigma}^{-1}}$ belongs to $L^{1}([0, T])$, Gronwall inequality implies that $\delta=0$ on $[0, T]$. Thus, uniqueness holds.

We turn to the existence result. To do so, let $r \in\left(0, \frac{1}{10}\right)$, such that

$$
\left(\left\|\left(u^{0}, b^{0}\right)\right\|_{\mathcal{X}_{a, \sigma}^{-1}}+r\right)^{1 / 2} r^{1 / 2}<\frac{1}{32}
$$


and $N \in \mathbb{N}$, such that

$$
\int_{|\xi|>N} \frac{e^{a|\xi|^{1 / \sigma}}}{|\xi|}\left|\hat{u^{0}}(\xi)\right| d \xi+\int_{|\xi|>N} \frac{e^{a|\xi|^{1 / \sigma}}}{|\xi|}\left|\hat{b^{0}}(\xi)\right| d \xi<\frac{r}{5} .
$$

Let $v^{0}=\mathcal{F}^{-1}\left(\mathbf{1}_{\{|\xi|<N\}} \hat{u^{0}}(\xi)\right)$ (respectively $c^{0}=\mathcal{F}^{-1}\left(\mathbf{1}_{\{|\xi|<N\}} \hat{b^{0}}(\xi)\right)$ ) the low frequencies part of $u^{0}$ (respectively $b^{0}$ ) and $w^{0}$ (respectively $\left.d^{0}\right)$ its high frequencies part corresponding to $(|\xi|>N)$. Clearly, one has

$$
\left\|\left(d^{0}, w^{0}\right)\right\|_{\mathcal{X}_{a, \sigma}^{-1}}<\frac{r}{5}
$$

By a standard Fourier computation, one infers that $(v, c)=e^{\nu t \Delta}\left(v^{0}, c^{0}\right)$ is the unique solution to the following linear system

$(M H D L)$

$$
\left\{\begin{array}{l}
\partial_{t} v-\Delta v=0, \quad(t, x) \in \mathbb{R}^{+} \times \mathbb{R}^{3} \\
\partial_{t} c-\Delta c=0, \quad(t, x) \in \mathbb{R}^{+} \times \mathbb{R}^{3} \\
\operatorname{div} v=0, \quad(t, x) \in \mathbb{R}^{+} \times \mathbb{R}^{3} \\
\operatorname{div} c=0, \quad(t, x) \in \mathbb{R}^{+} \times \mathbb{R}^{3} \\
(v, c)(0)=\left(v^{0}, c^{0}\right), \quad x \in \mathbb{R}^{3}
\end{array}\right.
$$

and that for all $t \geq 0$,

$$
\|(v, c)\|_{\mathcal{X}_{a, \sigma}^{-1}} \leq\left\|\left(u^{0}, b^{0}\right)\right\|_{\mathcal{X}_{a, \sigma}^{-1}} .
$$

By definition of $\mathcal{X}_{a, \sigma}^{1}$ norm, expression of $(v, c)$ and Tonelli's theorem, we infers that

$$
\|(v, c)\|_{L_{T}^{1}\left(\mathcal{X}_{a, \sigma}^{1}\right)} \leq \int_{\mathbb{R}^{3}}\left(1-e^{-\nu T|\xi|^{2}}\right)|\xi|^{-1} e^{a|\xi|^{1 / \sigma}}\left(\left|\hat{u^{0}}(\xi)\right|+\left|\hat{b^{0}}(\xi)\right|\right) d \xi
$$

Using the Dominated Convergence Theorem, we get

$$
\lim _{T \rightarrow 0^{+}}\|(v, c)\|_{L_{T}^{1}\left(\mathcal{X}_{a, \sigma}^{1}\right)}=0
$$

As it will be seen below, for instance, the stability condition of the fixed point argument requires a choice of $\varepsilon>0$ such that $\varepsilon^{1 / 2}\left\|u^{0}\right\|_{\mathcal{X}_{a, \sigma}^{-1}}^{1 / 2}<\frac{1}{18}$. Moreover, for the operator $\Psi$ to be a contraction mapping, $\varepsilon$ has to fulfill the supplementary condition $\left(\left\|\left(u^{0}, b^{0}\right)\right\|_{\mathcal{X}_{a, \sigma}^{-1}}+r\right)^{1 / 2} \varepsilon^{1 / 2}<\frac{1}{32}$. For this choice of $\varepsilon$, by (2.8), there exists a time $T=T(\varepsilon)>0$ such that

$$
\|v\|_{L_{T}^{1}\left(\mathcal{X}_{a, \sigma}^{1}\right)}<\varepsilon
$$


Put $w=u-v$ and $d=b-c$, the $3+3$ components vector $(w, d)$ satisfies, for all $(t, x)$, the following nonlinear system denoted (MHDNL),

$$
\left\{\begin{array}{l}
\partial_{t} w-\Delta w+(w+v) \cdot \nabla(w+v)-(d+c) \cdot \nabla(d+c)+\nabla\left(p+\frac{1}{2}|d+c|^{2}\right)=0 \\
\partial_{t} d-\Delta d+(w+v) \cdot \nabla(d+c)-(d+c) \cdot \nabla(w+v)=0 \\
\operatorname{div} w=0 \\
\operatorname{div} d=0 \\
(u, b)=\left(u^{0}, b^{0}\right) .
\end{array}\right.
$$

To run a fixed point argument, we introduce the following operator $\Psi$ defined for all $(w, d)^{T}$ by the right hand side of the following integral equation

$$
\left(\begin{array}{l}
w \\
d
\end{array}\right)=e^{\nu t \Delta}\left(\begin{array}{c}
w^{0} \\
d^{0}
\end{array}\right)-\int_{0}^{t} e^{\nu(t-\tau) \Delta}\left(\begin{array}{c}
(w+v) \cdot \nabla(w+v)-(d+c) \cdot \nabla(d+c) \\
(w+v) \cdot \nabla(d+c)-(d+c) \cdot \nabla(w+v)
\end{array}\right) d \tau,
$$

and we consider the space $\mathcal{X}_{T}:=\mathcal{C}\left([0, T],\left(\mathcal{X}_{a, \sigma}^{-1}\left(\mathbb{R}^{3}\right)\right)^{2}\right) \cap L^{1}\left([0, T],\left(\mathcal{X}_{a, \sigma}^{1}\left(\mathbb{R}^{3}\right)\right)^{2}\right)$, endowed with its naturel norm $\|f\|_{\mathcal{X}_{T}}:=\|f\|_{L_{T}^{\infty}\left(\left(\mathcal{X}_{a, \sigma}^{-1}\right)^{2}\right)}+\|f\|_{L_{T}^{1}\left(\left(\mathcal{X}_{a, \sigma}^{1}\right)^{2}\right)}$.

In a first step, let us prove that $\mathcal{X}_{T}$ is stable under the operator $\Psi$. To do so, we denote by $\mathbf{B}_{r}$ the subset of $\mathcal{X}_{T}$ defined by

$$
\mathbf{B}_{r}=\left\{(u, b) \in \mathcal{X}_{T} ;\|(u, b)\|_{L_{T}^{\infty}\left(\mathcal{X}_{a, \sigma}^{-1}\right)} \leq r ;\|(u, b)\|_{L_{T}^{1}\left(\mathcal{X}_{a, \sigma}^{1}\right)} \leq r\right\} .
$$

For $(w, d) \in \mathbf{B}_{r}$, we have $\psi((w, d)) \in \mathbf{B}_{r}$. In fact, it holds that

$$
\begin{aligned}
\|\Psi(w, d)(t)\|_{\mathcal{X}_{a, \sigma}^{-1}} & \leq I_{w w}+I_{w v}+I_{v w}+I_{v v}+I_{d d}+I_{d c}+I_{c d}+I_{c c} \\
& +I_{w d}+I_{w c}+I_{v d}+I_{v c}+I_{d w}+I_{d v}+I_{c w}+I_{c v}
\end{aligned}
$$

where we denoted, for any divergence free vector field $v$ and $\omega$,

$$
I_{v \omega}=\int_{0}^{t}\left\|e^{(t-\tau) \Delta} v \cdot \nabla \omega\right\|_{\mathcal{X}_{a, \sigma}^{-1}} d \tau
$$

To estimate $\|\Psi(w, d)(t)\|_{\mathcal{X}_{a, \sigma}^{-1}}$, we recall that according to the choice of $N$, we have

$$
\left\|w^{0}\right\|_{\mathcal{X}_{a, \sigma}^{-1}}+\left\|d^{0}\right\|_{\mathcal{X}_{a, \sigma}^{-1}}<\frac{r}{9}
$$

Using divergence free condition and lemma 1.5, we obtain that

$$
I_{v v} \leq\|v\|_{L_{T}^{\infty}\left(\mathcal{X}_{a, \sigma}^{-1}\right)}\|v\|_{L_{T}^{1}\left(\mathcal{X}_{a, \sigma}^{1}\right)} \leq \varepsilon\left\|u^{0}\right\|_{\mathcal{X}_{a, \sigma}^{-1}}<\frac{r}{18} .
$$

The same holds for $I_{c c}, I_{v c}$ and $I_{c v}$. Moreover,

$$
I_{w w} \leq\|w\|_{L_{T}^{\infty}\left(\mathcal{X}_{a, \sigma}^{-1}\right)}\|w\|_{L_{T}^{1}\left(\mathcal{X}_{a, \sigma}^{1}\right)} \leq r^{2}<\frac{r}{18}
$$

and the same holds for $I_{d d}, I_{w d}$ and $I_{d w}$. Furthermore,

$$
I_{v w} \leq\|v\|_{L_{T}^{\infty}\left(\mathcal{X}_{a, \sigma}^{-1}\right)}^{1 / 2}\|v\|_{L_{T}^{1}\left(\mathcal{X}_{a, \sigma}^{1}\right)}^{1 / 2}\|w\|_{L_{T}^{\infty}\left(\mathcal{X}_{a, \sigma}^{-1}\right)}^{1 / 2}\|w\|_{L_{T}^{1}\left(\mathcal{X}_{a, \sigma}^{1}\right)}^{1 / 2} \leq r \varepsilon^{1 / 2}\left\|u^{0}\right\|_{\mathcal{X}_{a, \sigma}^{-1}}^{1 / 2}<\frac{r}{18}
$$


and the same holds for the seven remaining integrals. Finally, we obtain

$$
\|\Psi(w, d)(t)\|_{\mathcal{X}_{a, \sigma}^{-1}} \leq r
$$

Let us estimate $\|\Psi(w)(t)\|_{L^{1}\left(\mathcal{X}_{a, \sigma}^{1}\right)}$. As above, we have

$$
\begin{aligned}
\|\Psi(w)(t)\|_{L^{1}\left(\mathcal{X}_{a, \sigma}^{1}\right)} & \leq J_{w w}+J_{w v}+J_{v w}+J_{v v}+J_{d d}+J_{d c}+J_{c d}+J_{c c} \\
& +J_{w d}+J_{w c}+J_{v d}+J_{v c}+J_{d w}+J_{d v}+J_{c w}+J_{c v}
\end{aligned}
$$

where we denoted, for any divergence free vector field $v$ and $\omega$,

$$
J_{v \omega}=\int_{0}^{T}\left\|\int_{0}^{t} e^{(t-\tau) \Delta} v \cdot \nabla \omega d \tau\right\|_{\mathcal{X}_{a, \sigma}^{1}} d t .
$$

By the facts that $\|v\|_{L_{T}^{1}\left(\mathcal{X}_{a, \sigma}^{1}\right)},\|c\|_{L_{T}^{1}\left(\mathcal{X}_{a, \sigma}^{1}\right)}<\varepsilon$, we can take

$$
\|v\|_{L_{T}^{1}\left(\mathcal{X}_{a, \sigma}^{1}\right)}+\|c\|_{L_{T}^{1}\left(\mathcal{X}_{a, \sigma}^{1}\right)}<\frac{r}{9} .
$$

Using lemma 1.6 and the fact that $w \in \mathbf{B}_{r}$, we get

$$
J_{v v} \leq\|v\|_{L_{T}^{\infty}\left(\mathcal{X}_{a, \sigma}^{-1}\right)}\|v\|_{L_{T}^{1}\left(\mathcal{X}_{a, \sigma}^{1}\right)} \leq \varepsilon\left\|u^{0}\right\|_{\mathcal{X}_{a, \sigma}^{-1}}<\frac{r}{18}
$$

and so on for $J_{c c}, J_{v c}$ and $J_{c v}$. Also, we get

$$
J_{w w} \leq\|w\|_{L_{T}^{\infty}\left(\mathcal{X}_{a, \sigma}^{-1}\right)}\|w\|_{L_{T}^{1}\left(\mathcal{X}_{a, \sigma}^{1}\right)} \leq r^{2}<\frac{r}{18}
$$

and so on for $J_{d d}, J_{w d}$ and $J_{d w}$. Moreover,

$$
J_{v w} \leq\|v\|_{L_{T}^{\infty}\left(\mathcal{X}_{a, \sigma}^{-1}\right)}^{1 / 2}\|v\|_{L_{T}^{1}\left(\mathcal{X}_{a, \sigma}^{1}\right)}^{1 / 2}\|w\|_{L_{T}^{\infty}\left(\mathcal{X}_{a, \sigma}^{-1}\right)}^{1 / 2}\|w\|_{L_{T}^{1}\left(\mathcal{X}_{a, \sigma}^{1}\right)}^{1 / 2} \leq r \varepsilon^{1 / 2}\left\|u^{0}\right\|_{\mathcal{X}_{a, \sigma}^{-1}}^{1 / 2}<\frac{r}{18}
$$

and so on for the seven remaining integrals. Thus,

$$
\|\Psi(w, d)(t)\|_{L^{1}\left(\mathcal{X}_{a, \sigma}^{1}\right)} \leq r
$$

Combining (2.11) and (2.13), we deduce that $\Psi\left(\mathbf{B}_{r}\right) \subset \mathbf{B}_{r}$.

In a second step, to prove that $\Psi$ is a contraction mapping on $\mathbf{B}_{r}$. One has

$$
\Psi\left(w_{2}, d_{2}\right)-\Psi\left(w_{1}, d_{1}\right)=-\int_{0}^{t} e^{(t-\tau) \Delta}\left(\begin{array}{c}
\alpha_{w w}+\alpha_{d d} \\
\alpha_{w d}+\alpha_{d w}
\end{array}\right) d \tau
$$

where

$$
\begin{aligned}
& \alpha_{w w}=\left(w_{2}+v\right) \cdot \nabla\left(w_{2}-w_{1}\right)-\left(w_{2}-w_{1}\right) \cdot \nabla\left(w_{1}+v\right) \\
& \alpha_{d d}=-\left(d_{2}+c\right) \cdot \nabla\left(d_{2}+c\right)+\left(d_{1}+c\right) \cdot \nabla\left(d_{1}+c\right) \\
& \alpha_{w d}=\left(w_{2}+v\right) \cdot \nabla\left(d_{2}+c\right)-\left(w_{1}+v\right) \cdot \nabla\left(d_{1}+c\right) \\
& \alpha_{d w}=-\left(d_{2}+c\right) \cdot \nabla\left(w_{2}+v\right)+\left(d_{1}+c\right) \cdot \nabla\left(w_{1}+v\right) .
\end{aligned}
$$


Or equivalently, in an adequate form to be estimated, one has

$$
\begin{aligned}
& \alpha_{w w}=\left(w_{2}+v\right) \cdot \nabla\left(w_{2}-w_{1}\right)-\left(w_{2}-w_{1}\right) \cdot \nabla\left(w_{1}+v\right) \\
& \alpha_{d d}=-\left(d_{2}+c\right) \cdot \nabla\left(d_{2}-d_{1}\right)+\left(d_{2}-d_{1}\right) \cdot \nabla\left(d_{1}+c\right) \\
& \alpha_{w d}=\left(w_{2}-w_{1}\right) \cdot \nabla\left(d_{2}+c\right)+\left(w_{1}-v\right) \cdot \nabla\left(d_{2}-d_{1}\right) \\
& \alpha_{d w}=-\left(d_{2}-d_{1}\right) \cdot \nabla\left(w_{2}+v\right)-\left(d_{1}+c\right) \cdot \nabla\left(w_{2}-w_{1}\right) .
\end{aligned}
$$

It follows that

$$
\left\|\Psi\left(\left(w_{2}, d_{2}\right)\right)-\Psi\left(\left(w_{1}, d_{1}\right)\right)\right\|_{\mathcal{X}_{a, \sigma}^{-1}} \leq \sum_{i=1}^{2} K_{w w}^{(i)}+K_{d d}^{(i)}+K_{w d}^{(i)}+K_{d d}^{(i)}
$$

where

$$
\begin{aligned}
& K_{w w}^{(1)}=\left\|\int_{0}^{t} e^{(t-\tau) \Delta}\left(w_{2}+v\right) \nabla\left(w_{2}-w_{1}\right) d \tau\right\|_{\mathcal{X}_{a, \sigma}^{-1}}, \\
& K_{w w}^{(2)}=\left\|\int_{0}^{t} e^{(t-\tau) \Delta}\left(w_{2}-w_{1}\right) \nabla\left(w_{1}+v\right) d \tau\right\|_{\mathcal{X}_{a, \sigma}^{-1}},
\end{aligned}
$$

and so on for the other integrals. Using lemma 1.5, triangle inequality, the fact that $w_{i}$ belongs to $B_{r}$, inequalities (2.7) and (2.9), we infer that

$$
\begin{array}{r}
\leq\left\|v+w_{2}\right\|_{L_{T}^{\infty}\left(\mathcal{X}_{a, \sigma}^{-1}\right)}^{1 / 2}\left\|v+w_{2}\right\|_{L_{T}^{1}\left(\mathcal{X}_{a, \sigma}^{1}\right)}^{1 / 2}\left\|w_{2}-w_{1}\right\|_{L_{T}^{\infty}\left(\mathcal{X}_{a, \sigma}^{-1}\right)}^{1 / 2}\left\|w_{2}-w_{1}\right\|_{L_{T}^{1}\left(\mathcal{X}_{a, \sigma}^{1}\right)}^{1 / 2}, K_{w w}^{(2)} \\
\leq\left(\left\|u^{0}\right\|_{\mathcal{X}_{a, \sigma}^{-1}}+r\right)^{1 / 2}(\varepsilon+r)^{1 / 2}\left\|w_{2}-w_{1}\right\|_{\mathcal{X}_{T}} \\
\leq\left(\left\|\left(u^{0}, b^{0}\right)\right\|_{\mathcal{X}_{a, \sigma}^{-1}}+r\right)^{1 / 2}(\varepsilon+r)^{1 / 2}\left\|w_{2}-w_{1}\right\|_{\mathcal{X}_{T}} .
\end{array}
$$

Similarly,

$$
\begin{aligned}
& K_{d d}^{(1)}, K_{d d}^{(2)} \leq\left(\left\|\left(u^{0}, b^{0}\right)\right\|_{\mathcal{X}_{a, \sigma}^{-1}}+r\right)^{1 / 2}(\varepsilon+r)^{1 / 2}\left\|d_{2}-d_{1}\right\|_{\mathcal{X}_{T}} \\
& K_{w d}^{(1)}, K_{d w}^{(2)} \leq\left(\left\|\left(u^{0}, b^{0}\right)\right\|_{\mathcal{X}_{a, \sigma}^{-1}}+r\right)^{1 / 2}(\varepsilon+r)^{1 / 2}\left\|w_{2}-w_{1}\right\|_{\mathcal{X}_{T}} \\
& K_{w d}^{(2)}, K_{d w}^{(1)} \leq\left(\left\|\left(u^{0}, b^{0}\right)\right\|_{\mathcal{X}_{a, \sigma}^{-1}}+r\right)^{1 / 2}(\varepsilon+r)^{1 / 2}\left\|d_{2}-d_{1}\right\|_{\mathcal{X}_{T}} .
\end{aligned}
$$

Thus,

$$
\begin{array}{r}
\left\|\Psi\left(w_{2}, d_{2}\right)-\Psi\left(w_{1}, d_{1}\right)\right\|_{L_{T}^{\infty}\left(\mathcal{X}_{a, \sigma}^{-1}\right)} \leq \\
4\left(\left\|\left(u^{0}, b^{0}\right)\right\|_{\mathcal{X}_{a, \sigma}^{-1}}+r\right)^{1 / 2}(\varepsilon+r)^{1 / 2}\left(\left\|w_{2}-w_{1}\right\|_{\mathcal{X}_{T}}+\left\|d_{2}-d_{1}\right\|_{\mathcal{X}_{T}}\right) .
\end{array}
$$

To estimate the $L_{T}^{1}\left(\mathcal{X}_{a, \sigma}^{1}\right)$ norm, we proceed as above;

$$
\left\|\Psi\left(\left(w_{2}, d_{2}\right)\right)-\Psi\left(\left(w_{1}, d_{1}\right)\right)\right\|_{L^{1}\left(\mathcal{X}_{a, \sigma}^{1}\right)} \leq \sum_{i=1}^{2} L_{w w}^{(i)}+L_{d d}^{(i)}+L_{w d}^{(i)}+L_{d d}^{(i)},
$$

where

$$
\begin{aligned}
L_{w w}^{(1)} & =\int_{0}^{T}\left\|\int_{0}^{t} e^{(t-\tau) \Delta}\left(w_{2}+v\right) \nabla\left(w_{2}-w_{1}\right) d \tau\right\|_{\mathcal{X}_{a, \sigma}^{1}} d t \\
L_{w w}^{(2)} & =\int_{0}^{T}\left\|\int_{0}^{t} e^{(t-\tau) \Delta}\left(w_{2}-w_{1}\right) \nabla\left(w_{1}+v\right) d \tau\right\|_{\mathcal{X}_{a, \sigma}^{1}} d t
\end{aligned}
$$


and so on for the other integrals. Using lemma 1.6, triangle inequality, the fact that $w_{i}$ belongs to $B_{r}$, inequalities (2.7) and (2.9), we infer that

$$
L_{w w}^{(1)}, L_{w w}^{(2)} \leq\left.\left(\left\|\left(u^{0}, b^{0}\right)\right\|_{\mathcal{X}_{a, \sigma}^{-1}}+r\right)^{1 / 2}(\varepsilon+r)^{1 / 2}\left\|w_{2}-w_{1}\right\|\right|_{\mathcal{X}_{T}}
$$

The same holds for $L_{d d}^{(i)}, L_{w d}^{(i)}$ and $L_{d w}^{(i)}$, and one obtains

$$
\begin{array}{r}
\left\|\Psi\left(w_{2}, d_{2}\right)-\Psi\left(w_{1}, d_{1}\right)\right\|_{L_{T}^{1}\left(\mathcal{X}_{a, \sigma}^{1}\right)} \leq \\
4\left(\left\|\left(u^{0}, b^{0}\right)\right\|_{\mathcal{X}_{a, \sigma}^{-1}}+r\right)^{1 / 2}(\varepsilon+r)^{1 / 2}\left(\left\|w_{2}-w_{1}\right\|_{\mathcal{X}_{T}}+\left\|d_{2}-d_{1}\right\|_{\mathcal{X}_{T}}\right) .
\end{array}
$$

By (2.14) and (2.15), we infer that

$$
\begin{array}{r}
\left\|\Psi\left(w_{2}, d_{2}\right)-\Psi\left(w_{1}, d_{1}\right)\right\|_{\mathcal{X}_{T}} \leq \\
8\left(\left\|\left(u^{0}, b^{0}\right)\right\|_{\mathcal{X}_{a, \sigma}^{-1}}+r\right)^{1 / 2}(\varepsilon+r)^{1 / 2}\left(\left\|w_{2}-w_{1}\right\|_{\mathcal{X}_{T}}+\left\|d_{2}-d_{1}\right\|_{\mathcal{X}_{T}}\right) .
\end{array}
$$

This implies that

$$
\left\|\Psi\left(w_{2}, d_{2}\right)-\Psi\left(w_{1}, d_{1}\right)\right\|_{\mathcal{X}_{T}} \leq \frac{1}{2}\left(\left\|w_{2}-w_{1}\right\|_{\mathcal{X}_{T}}+\left\|d_{2}-d_{1}\right\|_{\mathcal{X}_{T}}\right)
$$

and $\Psi$ is a contraction mapping. The fixed point theorem implies that there is a unique $(w, d) \in \mathbf{B}_{r}$, such that $(u, b)=(v+w, c+d)$ is the solution of $(M H D)$ with $(u, b) \in \mathcal{X}_{T}$.

\section{BlOWUP RESUlts}

In this section we prove theorem 0.2. First of all, the following energy estimates holds in $\mathcal{X}_{a, \sigma}^{-1}$,

$$
\begin{aligned}
\|(u, b)(t)\|_{\mathcal{X}_{a, \sigma}^{-1}}+\int_{0}^{t}\|(u, b)(t)\|_{\mathcal{X}_{a, \sigma}^{1}} & \leq\left\|\left(u_{0}, b_{0}\right)\right\|_{\mathcal{X}_{a, \sigma}^{-1}} \\
& +\int_{0}^{t} \mathcal{L}_{u u}(\tau)+\mathcal{L}_{b b}(\tau)+\mathcal{L}_{u b}(\tau)+\mathcal{L}_{b u}(\tau) d \tau
\end{aligned}
$$

where $\mathcal{L}_{v \omega}=\|\operatorname{div}(v \omega)\|_{\mathcal{X}_{a, \sigma}^{-1}}$. By lemma 1.2, we have

$$
\begin{aligned}
\mathcal{L}_{v \omega} & \leq\|v\|_{\mathcal{X}_{a, \sigma}^{0}}\|\omega\|_{\mathcal{X}_{\frac{a}{\sigma}, \sigma}^{0}}+\|\omega\|_{\mathcal{X}_{a, \sigma}^{0}}\|v\|_{\mathcal{X}_{\frac{a}{\sigma}, \sigma}^{0}} \\
& \leq 2\|(v, \omega)\|_{\mathcal{X}_{a, \sigma}^{0}}\|(u, b)\|_{\mathcal{X}_{\frac{a}{\sigma}, \sigma}^{0}} .
\end{aligned}
$$

It follows that

$$
\|(u, b)(t)\|_{\mathcal{X}_{a, \sigma}^{-1}}+\int_{0}^{t}\|(u, b)(t)\|_{\mathcal{X}_{a, \sigma}^{1}} \leq\left\|\left(u_{0}, b_{0}\right)\right\|_{\mathcal{X}_{a, \sigma}^{-1}}+8 \int_{0}^{t}\|(u, b)\|_{\mathcal{X}_{\frac{\alpha}{\sigma}, \sigma}^{0}}\|(u, b)\|_{\mathcal{X}_{a, \sigma}^{0}} d \tau .
$$

Using Lemma 1.3 and product Young inequality, we obtain

$$
\begin{aligned}
\|(u, b)(t)\|_{\mathcal{X}_{a, \sigma}^{-1}} & +\frac{1}{2} \int_{0}^{t}\|(u, b)(z)\|_{\mathcal{X}_{a, \sigma}^{1}} d z \leq\left\|\left(u_{0}, b_{0}\right)\right\|_{\mathcal{X}_{a, \sigma}^{-1}} \\
& +32 \int_{0}^{t}\|(u, b)\|_{\mathcal{X}_{\frac{a}{\sigma}, \sigma}^{0}}^{2}\|(u, b)\|_{\mathcal{X}_{a, \sigma}^{-1}} d \tau
\end{aligned}
$$


However, a direct computation implies that $\|(u, b)\|_{\mathcal{X}_{\frac{\alpha}{\sigma}, \sigma}^{0}} \leq M_{0}\|(u, b)\|_{\mathcal{X}_{a, \sigma}^{-1}}$, where the constant $M=$ $M_{0}(a, \sigma)=\sup _{r \geq 0} r e^{-a\left(\frac{1}{\sigma}-1\right) r^{1 / \sigma}}$. Then, estimation (3.1) becomes

$$
\|(u, b)(t)\|_{\mathcal{X}_{a, \sigma}^{-1}}+\frac{1}{2} \int_{0}^{t}\|(u, b)(z)\|_{\mathcal{X}_{a, \sigma}^{1}} d z \leq\left\|\left(u_{0}, b_{0}\right)\right\|_{\mathcal{X}_{a, \sigma}^{-1}}+32 M_{0}^{2} \int_{0}^{t}\|(u, b)\|_{\mathcal{X}_{a, \sigma}^{-1}}^{3} d z
$$

Let $T=\sup \left\{t \in\left[0, T^{*}\left[; \sup _{z \in[0, t]}\|(u, b)(z)\|_{\mathcal{X}_{a, \sigma}^{-1}} \leq 2\left\|\left(u_{0}, b_{0}\right)\right\|_{\mathcal{X}_{a, \sigma}^{-1}}\right\}\right.\right.$. By continuity of $(u, b)$, we have $T \in$ ] $\left.0, T^{*}\right]$ and

$$
2\left\|\left(u_{0}, b_{0}\right)\right\|_{\mathcal{X}_{a, \sigma}^{-1}} \leq\left\|\left(u_{0}, b_{0}\right)\right\|_{\mathcal{X}_{a, \sigma}^{-1}}+128 M_{0}^{2} T\left\|\left(u_{0}, b_{0}\right)\right\|_{\mathcal{X}_{a, \sigma}^{-1}}^{3}
$$

We infer that

$$
\frac{\left(128 M_{0}^{2}\right)^{-1}}{\left\|\left(u_{0}, b_{0}\right)\right\|_{\mathcal{X}_{a, \sigma}^{-1}}^{2}} \leq T \leq T^{*} .
$$

If we consider the magnetohydrodynamic system starting at initial time $t$, with the data $(u, b)(t)$, we get

$$
\frac{\left(128 M_{0}^{2}\right)^{-1}}{\|(u, b)(t)\|_{\mathcal{X}_{a, \sigma}^{-1}}^{2}} \leq T^{*}-t
$$

or equivalently

$$
\frac{\left(128 M_{0}^{2}\right)^{-1}}{T^{*}-t} \leq\|(u, b)(t)\|_{\mathcal{X}_{a, \sigma}^{-1}}^{2}
$$

Therefore, we infers that

$$
\lim _{t \rightarrow T^{*}}\|(u, b)(t)\|_{\mathcal{X}_{a, \sigma}^{-1}}=+\infty
$$

Applying Gronwall inequality to inequality (3.1), we get

$$
\|(u, b)(t)\|_{\mathcal{X}_{a, \sigma}^{-1}}+\frac{1}{2} \int_{0}^{t}\|(u, b)(t)\|_{\mathcal{X}_{a, \sigma}^{1}} \leq\left\|\left(u_{0}, b_{0}\right)\right\|_{\mathcal{X}_{a, \sigma}^{-1}} \exp \left(32 \int_{0}^{t}\|(u, b)\|_{\mathcal{X}_{\frac{a}{\sigma}, \sigma}^{0}}^{2} d z\right)
$$

By equation (3.2), we obtain

$$
\int_{0}^{T^{*}}\|(u, b)\|_{\mathcal{X}_{\frac{\alpha}{\sigma}, \sigma}^{0}}^{2} d z=\infty
$$

This implies that

$$
\lim _{t \rightarrow T^{*}}\|(u, b)(t)\|_{\mathcal{X}_{\frac{a}{\sigma}, \sigma}^{0}}=+\infty .
$$

At this point, we proved that $\mathcal{X}_{\frac{\alpha}{\sigma}, \sigma}^{0}$ norm of the solution blows up, in finite time.

Let $a^{\prime}=\frac{a}{\sigma} \in(0, a)$, using the same method, we obtain

$$
\begin{aligned}
\|(u, b)(t)\|_{\mathcal{X}_{a^{\prime}, \sigma}^{0}} & +\int_{0}^{t}\|(u, b)(t)\|_{\mathcal{X}_{a^{\prime}, \sigma}^{2}} \\
& \leq\left\|\left(u_{0}, b_{0}\right)\right\|_{\mathcal{X}_{a^{\prime}, \sigma}^{0}}+\int_{0}^{t} R_{u u}(z)+R_{b b}(z)+R_{u b}(z)+R_{b u}(z) d z,
\end{aligned}
$$

where $R_{x y}(t)=\|x . \nabla y\|_{\mathcal{X}_{a^{\prime}, \sigma}^{0}}$. Using Lemma 1.4, we get

$$
\begin{aligned}
R_{x y}(t) & \leq\|x\|_{\mathcal{X}_{a^{\prime}, \sigma}^{0}}\|y\|_{\mathcal{X}_{a^{\prime}, \sigma}^{1}} \\
& \leq\|(x, y)\|_{\mathcal{X}_{a^{\prime}, \sigma}^{0}}\|(x, y)\|_{\mathcal{X}_{a^{\prime}, \sigma}^{1}}
\end{aligned}
$$


It follows that

$$
\|(u, b)(t)\|_{\mathcal{X}_{a^{\prime}, \sigma}^{0}}+\int_{0}^{t}\|(u, b)(t)\|_{\mathcal{X}_{a^{\prime}, \sigma}^{2}} \leq\left\|\left(u_{0}, b_{0}\right)\right\|_{\mathcal{X}_{a^{\prime}, \sigma}^{0}}+4 \int_{0}^{t}\|(u, b)\|_{\mathcal{X}_{a^{\prime}, \sigma}^{0}}\|(u, b)\|_{\mathcal{X}_{a^{\prime}, \sigma}^{1}} d z .
$$

By lemma 1.3, we obtain

$$
\|(u, b)(t)\|_{\mathcal{X}_{a^{\prime}, \sigma}^{0}}+\int_{0}^{t}\|(u, b)\|_{\mathcal{X}_{a^{\prime}, \sigma}^{2}} \leq\left\|\left(u_{0}, b_{0}\right)\right\|_{\mathcal{X}_{a^{\prime}, \sigma}^{0}}+4 \int_{0}^{t}\|(u, b)\|_{\mathcal{X}_{a^{\prime}, \sigma}^{0}}^{3 / 2}\|(u, b)\|_{\mathcal{X}_{a^{\prime}, \sigma}^{2}}^{1 / 2} .
$$

Using product Young inequality, we get

$$
\|(u, b)(t)\|_{\mathcal{X}_{a^{\prime}, \sigma}^{0}}+\frac{1}{2} \int_{0}^{t}\|(u, b)\|_{\mathcal{X}_{a^{\prime}, \sigma}^{2}} \leq\left\|\left(u_{0}, b_{0}\right)\right\|_{\mathcal{X}_{a^{\prime}, \sigma}^{0}}+4 \int_{0}^{t}\|(u, b)\|_{\mathcal{X}_{a^{\prime}, \sigma}^{0}}^{3} .
$$

Gronwall lemma gives

$$
\|(u, b)(t)\|_{\mathcal{X}_{a^{\prime}, \sigma}^{0}} \leq\left\|\left(u_{0}, b_{0}\right)\right\|_{\mathcal{X}_{a^{\prime}, \sigma}^{0}} \exp \left(4 \int_{0}^{t}\|(u, b)\|_{\mathcal{X}_{a^{\prime}, \sigma}^{0}}^{2} d z\right)
$$

Or equivalently,

$$
8\|(u, b)(t)\|_{\mathcal{X}_{a^{\prime}, \sigma}^{0}}^{2} \exp \left(-8 \int_{0}^{t}\|(u, b)\|_{\mathcal{X}_{a^{\prime}, \sigma}^{0}}^{2} d z\right) \leq 8\left\|\left(u_{0}, b_{0}\right)\right\|_{\mathcal{X}_{a^{\prime}, \sigma}^{0}}^{2}
$$

Integrating over $\left[0, T^{*}\right)$ and using (3.4), we infer that

$$
1 \leq 8\left\|\left(u_{0}, b_{0}\right)\right\|_{\mathcal{X}_{a^{\prime}, \sigma}^{0}}^{2} T^{*}
$$

Since $\frac{a}{\sigma^{n}}<\ldots<\frac{a}{\sigma}<a$, by the same method we used for $a^{\prime} \in(0, a)$, we prove that

$$
1 \leq 8\left\|\left(u_{0}, b_{0}\right)\right\|_{\mathcal{X}_{\frac{a}{\sigma^{n}}, \sigma}^{0}}^{2} T^{*}, \forall n \in \mathbb{N}^{*}
$$

By Dominate Convergence Theorem, we obtain

$$
\frac{1}{2 \sqrt{2} \sqrt{T^{*}}} \leq\left\|\left(u_{0}, b_{0}\right)\right\|_{\mathcal{X}^{0}}
$$

Consider the $(M H D)$ system starting at $t \in\left[0, T^{*}\right)$, by time translation, we have

$$
\frac{1}{2 \sqrt{2} \sqrt{T^{*}-t}} \leq\|(u, b)(t)\|_{\mathcal{X}^{0}} .
$$

At this point, we proved that the $\mathcal{X}^{0}$ norm of the solution blows up, in finite time.

Let $k \in \mathbb{N}^{*}$, we consider the subset $\mathcal{A}_{k}$ defined by

$$
\mathcal{A}_{k}=\left\{\xi \in \mathbb{R}^{3} ;|\xi| \leq k \text { and }\left|\widehat{u^{0}}(\xi)\right| \leq k\right\}
$$

and $v^{0}$ and $c^{0}$ in $L^{2}\left(\mathbb{R}^{3}\right) \cap \mathcal{X}_{a, \sigma}^{-1}\left(\mathbb{R}^{3}\right)$, such that $\left(v_{k}^{0}, c_{k}^{0}\right)=\mathcal{F}^{-1}\left(1_{\mathcal{A}_{k}}(\xi)\left(\widehat{\left(u^{0}\right.}, \widehat{b^{0}}\right)\right)$. Let $\left(w_{k}^{0}, d_{k}^{0}\right)=\left(u^{0}-v_{k}^{0}, b^{0}-\right.$ $\left.c_{k}^{0}\right)$, one has $\lim _{k \rightarrow \infty}\left\|\left(w_{k}^{0}, d_{k}^{0}\right)\right\|_{\mathcal{X}_{a, \sigma}^{-1}}=0$. So, there exists $k \in \mathbb{N}$, such that $\left\|\left(w_{k}^{0}, d_{k}^{0}\right)\right\|_{\mathcal{X}_{a, \sigma}^{-1}}<\frac{1}{16}$. Using 
smallness theory, we prove that a unique and global in time solution $\left(w_{k}, d_{k}\right)$ to the system

$\left(M H D_{1}\right)$

$$
\left\{\begin{array}{l}
\partial_{t} w-\Delta w+w \cdot \nabla w-d \cdot \nabla d=-\nabla \pi_{1}, \quad \text { in } \mathbb{R}^{+} \times \mathbb{R}^{3} \\
\partial_{t} d-\Delta d+w \cdot \nabla d-d \cdot \nabla w=0, \quad \text { in } \mathbb{R}^{+} \times \mathbb{R}^{3} \\
\operatorname{div} w=\operatorname{div} d=0, \quad \text { in } \mathbb{R}^{+} \times \mathbb{R}^{3} \\
(w, d)(0, x)=\left(w_{k}^{0}, d_{k}^{0}\right)(x), \quad \text { in } \mathbb{R}^{3}
\end{array}\right.
$$

exists in $C_{b}\left(\mathbb{R}^{+}, \mathcal{X}_{a, \sigma}^{-1}\left(\mathbb{R}^{3}\right)\right) \cap L^{1}\left(\mathbb{R}^{+}, \mathcal{X}_{a, \sigma}^{1}\left(\mathbb{R}^{3}\right)\right)$ and satisfies for $t \geq 0$

$$
\left\|\left(w_{k}, d_{k}\right)(t)\right\|_{\mathcal{X}_{a, \sigma}^{-1}}+\frac{1}{2} \int_{0}^{t}\left\|\left(w_{k}, d_{k}\right)(z)\right\|_{\mathcal{X}_{a, \sigma}^{1}} d z \leq\left\|\left(w_{k}^{0}, d_{k}^{0}\right)\right\|_{\mathcal{X}_{a, \sigma}^{-1}} .
$$

Consider $\left(v_{k}, c_{k}\right)=\left(u-w_{k}, b-c_{k}\right)$, it belongs to $C\left(\left[0, T^{*}\right), \mathcal{X}_{a, \sigma}^{-1}\left(\mathbb{R}^{3}\right)\right)$ and satisfies, for all $(t, x) \in \mathbb{R} \times \mathbb{R}^{3}$, the following $\left(\mathrm{MHD}_{2}\right)$ system,

$$
\left\{\begin{array}{l}
\partial_{t} v_{k}-\Delta v_{k}+v_{k} \cdot \nabla v_{k}+v_{k} \cdot \nabla w_{k}+w_{k} \cdot \nabla v_{k}-c_{k} \cdot \nabla c_{k}-c_{k} \cdot \nabla d_{k}-d_{k} \cdot \nabla c_{k}=-\nabla \pi_{2} \\
\partial_{t} c_{k}-\Delta c_{k}+v_{k} \cdot \nabla c_{k}+v_{k} \cdot \nabla d_{k}+w_{k} \cdot \nabla c_{k}-c_{k} \cdot \nabla v_{k}-c_{k} \cdot \nabla w_{k}-d_{k} \cdot \nabla v_{k}=0 \\
\operatorname{div} w=\operatorname{div} d=0 \\
\left(v_{k}, c_{k}\right)(0, x)=\left(v_{k}^{0}, c_{k}^{0}\right)(x)
\end{array}\right.
$$

Having that $\left(v_{k}^{0}, c_{k}^{0}\right) \in L^{2}\left(\mathbb{R}^{3}\right)$, we take the scalar product and use $L^{2}$ theory. Under divergence free condition, we infers that

$$
\frac{1}{2} \frac{d}{d t}\left\|\left(v_{k}, c_{k}\right)(t)\right\|_{L^{2}}^{2}+\left\|\left(\nabla v_{k}, \nabla c_{k}\right)(t)\right\|_{L^{2}}^{2} \leq C\left\|\left(d_{k}, w_{k}\right)\right\|_{L^{\infty}}^{2}\left\|\left(v_{k}, c_{k}\right)(t)\right\|_{L^{2}}^{2}
$$

The Gronwall lemma implies that

$$
\left\|\left(v_{k}, c_{k}\right)(t)\right\|_{L^{2}}^{2}+\int_{0}^{t}\left\|\left(\nabla v_{k}, \nabla c_{k}\right)(z)\right\|_{L^{2}}^{2} d z \leq\left\|\left(v_{k}^{0}, c_{k}^{0}\right)\right\|_{L^{2}}^{2} \exp \left(C \int_{0}^{t}\left\|\left(w_{k}, d_{k}\right)\right\|_{L^{\infty}}^{2} d z\right) .
$$

Using that $\|f\|_{L^{\infty}} \leq\|\widehat{f}\|_{L^{1}}=\|f\|_{\mathcal{X}^{0}} \leq\|f\|_{\mathcal{X}^{-1}}^{1 / 2}\|f\|_{\mathcal{X}^{1}}^{1 / 2}$, we obtain

$$
\begin{aligned}
\left\|\left(v_{k}, c_{k}\right)(t)\right\|_{L^{2}}^{2} & +\int_{0}^{t}\left\|\left(\nabla v_{k}, \nabla c_{k}\right)(z)\right\|_{L^{2}}^{2} d z \\
& \leq\left\|\left(v_{k}^{0}, c_{k}^{0}\right)\right\|_{L^{2}}^{2} e^{C} \int_{0}^{t}\left\|\left(w_{k}, d_{k}\right)\right\|_{\mathcal{X}^{-1}}\left\|\left(w_{k}, d_{k}\right)\right\|_{\mathcal{X}^{1}} d z \\
& \leq\left\|\left(v_{k}^{0}, c_{k}^{0}\right)\right\|_{L^{2}}^{2} e^{C} \int_{0}^{t}\left\|\left(w_{k}, d_{k}\right)\right\|_{\mathcal{X}_{a, \sigma}^{-1}}\left\|\left(w_{k}, d_{k}\right)\right\|_{\mathcal{X}_{a, \sigma}^{1}} d z
\end{aligned}
$$

By the energy estimates (3.6), we obtain the following $L^{2}$ energy estimates

$$
\left\|\left(v_{k}, c_{k}\right)(t)\right\|_{L^{2}}^{2}+\int_{0}^{t}\left\|\left(\nabla v_{k}, \nabla c_{k}\right)(z)\right\|_{L^{2}}^{2} d z \leq \alpha_{0}
$$

where $\alpha_{0}=\left\|\left(v_{k}^{0}, c_{k}^{0}\right)\right\|_{L^{2}}^{2} \exp \left(2 C\left\|\left(w_{k}^{0}, d_{k}^{0}\right)\right\|_{\mathcal{X}_{a, \sigma}^{-1}}^{2}\right)$. At this point, thanks to the properties of small frequencies part in $\mathcal{X}_{a, \sigma}^{-1}$, we closed the $L^{2}$ energy estimate of the large frequencies part of the solution. Using $\mathcal{X}_{a, \sigma}^{-1}$ 
energy estimates (3.6), we obtain

$$
\begin{aligned}
\|(u, b)\|_{\mathcal{X}^{0}} & \leq\left\|\left(v_{k}, c_{k}\right)\right\|_{\mathcal{X}^{0}}+\left\|\left(w_{k}, d_{k}\right)\right\|_{\mathcal{X}^{0}} \\
& \leq\left\|\left(v_{k}, c_{k}\right)\right\|_{\mathcal{X}^{0}}+M_{1}\left\|\left(w_{k}, d_{k}\right)\right\|_{\mathcal{X}_{a, \sigma}^{-1}} \\
& \leq\left\|\left(v_{k}, c_{k}\right)\right\|_{\mathcal{X}^{0}}+M_{1}\left\|\left(w_{k}^{0}, d_{k}^{0}\right)\right\|_{\mathcal{X}_{a, \sigma}^{-1}},
\end{aligned}
$$

where $M_{1}=M_{1}(a, \sigma)=\sup _{r \geq 0} r e^{-a r^{1 / \sigma}}$.

Inequality (3.5) implies that the function $t \rightarrow\|(u, b)(t)\|_{\mathcal{X}^{0}}$ is continuous on $\left[0, T^{*}\right)$ and tends to infinity when $t$ approaches $T^{*}$. Thus, there is $T_{0} \in\left[0, T^{*}\right)$, such that

$$
\frac{1 / 4}{\left(T^{*}-t\right)^{1 / 2}} \leq\left\|\left(v_{k}, c_{k}\right)(t)\right\|_{\mathcal{X}^{0}}, \forall t \in\left[T_{0}, T^{*}\right)
$$

At this point, we proved that the high frequencies part considered above blows up in the $\mathcal{X}^{0}$ norm. Thus, according to equation (3.5), one can infers that only high frequencies are responsible for this phenomena. Now, using Lemma 1.1 and (3.8), we can involve $\mathcal{X}^{s}$. Mainly, for $s \geq 3 / 2$ and $t \in\left[T_{0}, T^{*}\right)$,

$$
\frac{1 / 4}{\left(T^{*}-t\right)^{1 / 2}} \leq\left\|\left(v_{k}, c_{k}\right)(t)\right\|_{L^{2}}^{\frac{s}{s+\frac{3}{2}}}\left\|\left(v_{k}, c_{k}\right)(t)\right\|_{\mathcal{X}^{s}}^{\frac{\frac{3}{2}}{s+\frac{3}{2}}}
$$

Using inequality (3.7), we can omit the $L^{2}$ norm and obtain

$$
\frac{1 / 4}{\left(T^{*}-t\right)^{1 / 2}} \leq \alpha_{0}^{\frac{s}{s+\frac{3}{2}}}\left\|\left(v_{k}, c_{k}\right)(t)\right\|_{\mathcal{X}^{s}}^{\frac{\frac{3}{2}}{s+\frac{3}{2}}}
$$

This implies for $n \in \mathbb{N}$, such that $\frac{n}{\sigma}-1 \geq 3 / 2$ or $n \geq n_{0}=\left[\frac{5}{2} \sigma\right]+1$,

$$
\frac{4^{-1 / 3}}{\left(T^{*}-t\right)^{1 / 3}} \frac{a^{n}}{n !}\left(\frac{4^{-\frac{2}{3 \sigma}} \alpha_{0}^{-\frac{2}{3 \sigma}}}{\left(T^{*}-t\right)^{\frac{1}{3 \sigma}}}\right)^{n} \leq \frac{a^{n}}{n !}\left\|\left(v_{k}, c_{k}\right)(t)\right\|_{\mathcal{X}^{\frac{n}{\sigma}-1}}
$$

Summing up for $n \geq n_{0}$, we get

$$
\begin{aligned}
\frac{4^{-1 / 3}}{\left(T^{*}-t\right)^{1 / 3}} \sum_{n \geq n_{0}} \frac{a^{n}}{n !}\left(\frac{4^{-\frac{2}{3 \sigma}} \alpha_{0}^{-\frac{2}{3 \sigma}}}{\left(T^{*}-t\right)^{\frac{1}{3 \sigma}}}\right)^{n} & \leq \sum_{n \geq n_{0}} \frac{a^{n}}{n !}\left\|\left(v_{k}, c_{k}\right)(t)\right\|_{\mathcal{X}^{\frac{n}{\sigma}-1}} \\
& \leq\left\|\left(v_{k}, c_{k}\right)(t)\right\|_{\mathcal{X}_{a, \sigma}^{-1}}
\end{aligned}
$$

Using triangular inequality and $\mathcal{X}_{a, \sigma}^{-1}$ energy estimates (3.6), we have

$$
\left\|\left(v_{k}, c_{k}\right)(t)\right\|_{\mathcal{X}_{a, \sigma}^{-1}} \leq\|(u, b)(t)\|_{\mathcal{X}_{a, \sigma}^{-1}}+\left\|\left(w_{k}^{0}, d_{k}^{0}\right)\right\|_{\mathcal{X}_{a, \sigma}^{-1}}
$$

Dividing both sides of the resulting inequality by $\exp \left(a \frac{4^{-\frac{2}{3 \sigma}} \alpha_{0}^{-\frac{2}{3 \sigma}}}{\left(T^{*}-t\right)^{\frac{1}{3 \sigma}}}\right)$, we infer that

$$
\liminf _{t \rightarrow T^{*}}\left(T^{*}-t\right)^{1 / 3} \exp \left(-\frac{4^{-\frac{2}{3 \sigma}} \alpha_{0}^{-\frac{2}{3 \sigma}}}{\left(T^{*}-t\right)^{\frac{1}{3 \sigma}}}\right)\|(u, b)(t)\|_{\mathcal{X}_{a, \sigma}^{-1}} \geq 4^{-1 / 3}
$$

This gives the blowup profile and finishes the proof. 
Remark 3.1. Let $U^{0}=\left(u^{0}, b^{0}\right) \in \mathcal{X}_{a, \sigma}^{-1}\left(\mathbb{R}^{3}\right)$, where $a>0, \sigma \geq 1$. Let $U=(u, b)$ be the maximal solution of (MHD) system. Using the fact

$$
\mathcal{X}_{a, \sigma}^{-1}\left(\mathbb{R}^{3}\right) \hookrightarrow \mathcal{X}_{a^{\prime}, \sigma}^{-1}\left(\mathbb{R}^{3}\right) \hookrightarrow \mathcal{X}^{-1}\left(\mathbb{R}^{3}\right), \forall 0<a^{\prime}<a,
$$

one infers that $U \in C\left(\left[0, T_{a, \sigma}^{*}\right), \mathcal{X}_{a, \sigma}^{-1}\left(\mathbb{R}^{3}\right)\right), U \in C\left(\left[0, T_{a^{\prime}, \sigma}^{*}\right), \mathcal{X}_{a^{\prime}, \sigma}^{-1}\left(\mathbb{R}^{3}\right)\right)$ and $U \in C\left(\left[0, T^{*}\right), \mathcal{X}^{-1}\left(\mathbb{R}^{3}\right)\right)$, where the maximal times of existence $T_{a, \sigma}^{*}, T_{a^{\prime}, \sigma}^{*}$ and $T^{*}$ belong all of them to $(0,+\infty]$. These times satisfy $T_{a, \sigma}^{*} \leq$ $T_{a^{\prime}, \sigma}^{*} \leq T^{*}$. By the method we used in the proof of the blowup result $\left(a^{\prime}=\frac{a}{\sigma^{n}}\right)$, we proved that $T_{a, \sigma}^{*}=T^{*}$, if $\sigma>1$. However, we note that if $\sigma=1$ our technics failes. So, this critical case seems to need an other approach different from us.

Remark 3.2. Using the same technics we can prove existence, uniqueness and blowup results in finite time of solution to the periodic MHD, the three dimensional Navier-Stokes system, the two dimensional quasi-geostrophic equations with subcritical dissipation, in the Lei-Lin-Gevrey space.

Acknowledgement: The authors gratefully acknowledge the approval and the support of this research study by the grant number SCI-2017-1-7-F-7081 from the Deanship of Scientific Research at Northern Border University, Arar, K. S. A.

Conflicts of Interest: The author(s) declare that there are no conflicts of interest regarding the publication of this paper.

\section{REFERENCES}

[1] J. T. Beale, T. Kato and A. Majda, Remarks on the breakdown of smooth solutions for the 3-D Euler equations, Commun. Math. Phys. 94 (1984), 61—66.

[2] J. Benameur, On the blow-up criterion of 3D Navier-Stokes equations, J. Math. Anal. Appl. 371 (2010), $719--727$.

[3] J. Benameur, On the blow-up criterion of the periodic incompressible fluids, Math. Methods Appl. Sci. 36 (2) (2013), 143-153.

[4] J. Benameur, On the exponential type explosion of Navier-Stokes equations, Nonlinear Anal., Theory Methods Appl. 103 (2014), 87-97.

[5] J. Benameur and L. Jlali, On the blow-up criterion of 3D-NSE in Sobolev-Gevrey spaces, J. Math. Fluid Mech. 18 (4) (2016), 805-822.

[6] J. Benameur and R. Selmi, Anisotropic Rotating MHD System in Critical Anisotropic Spaces, Mem. Differential Equations Math. Phys. 44 (2008), 23-44.

[7] J. Benameur and R. Selmi, Study of anisotropic MHD system in anisotropic Sobolev spaces, Ann. Fac. Sci. Toulouse Math., 17 (1) (2008), 1-22.

[8] J. Benameur and R. Selmi, Long time decay to the Leray solution of the two-dimensional Navier-Stokes equations, Bull. London Math. Soc. 44 (5) (2012), 1001-1019.

[9] J. Benameur and R. Selmi, Time decay and exponential stability of solutions to the periodic 3D Navier-Stokes equation in critical spaces, Math. Methods Appl. Sci. 37 (17) (2014), 2817-2828. 
[10] M. Cannone, Harmonic analysis tools for solving the incompressible Navier-Stokes equations, Handbook of Mathematical Fluid Dynamics, vol 3, eds. S. Friedlander and D. Serre, Elsevier, 2003.

[11] J-Y. Chemin, Remarques sur l'existence global pour le systeme de Navier-Stokes incompressible, SIAM J. Math. Anal. 23 (1) (1992), 27-50.

[12] H. Fujita and T. Kato, On the Navier-Stokes initial value problem I, Arch. Ration. Mech. Anal. 16 (1964), $269-315$.

[13] T. Kato, Strong $L^{p}$ solutions of the Navier-Stokes equations in $\mathbb{R}^{m}$ with applications to weak solutions, Math. Z. 187 (4)(1984), 471-480.

[14] H. Koch and D. Tataru, Well-posedness for the Navier-Stokes equations, Adv. Math. 157 (1) (2001), 22-35.

[15] Z. Lei and F. Lin, Global mild solutions of Navier-Stokes equations, Commun. Pure Appl. Math. 64 (2011), $1297-1304$.

[16] Z. Lei and L. Xiuting, The local well-posedness, blow-up criteria and Gevrey regularity of solutions for a two-component high-order Camassa-Holm system, Nonlinear Anal., Real. World Appl. 35 (2017), 414-440.

[17] J. Leray, Essai sur le mouvement d'un liquide visqueux emplissant l'espace, Acta Math. 63 (1933), 22-25.

[18] R. Selmi, Convergence results for MHD system, Internat. J. Math. Math. Sci. 2006 (2006), Article ID 28704.

[19] R. Selmi, Asymptotic study of mixed rotating MHD system, Bull. Korean Math. Soc. 47 (2) (2010), 231-250.

[20] R. Selmi, Global well-posedness and convergence results for 3D-regularized Boussinesq system, Canad. J. Math. 64 (6) (2012), 1415-1435. 